\title{
Wind-driven Exclusion of Cosmic Rays in the Protoplanetary Disk Environment
}

\author{
L. Ilsedore Cleeves*, Edwin A. Bergin, Fred C. Adams \\ University of Michigan \\ E-mail: cleeves@umich.edu
}

\begin{abstract}
The recent (apparent) passage of the Voyager 1 spacecraft into interstellar space provides us with front-row seats to the complex interplay between the solar wind and the protective surrounding bubble known as heliosphere. The heliosphere extends radially out to $\sim 100 \mathrm{AU}$ from the sun, and within this sphere of influence, the solar wind modulates the incoming flux of galactic cosmic rays (CRs), especially those at low energies. Newly formed stars, which support both strong magnetic fields and winds, are expected to produce analogous regions of CR exclusion, perhaps at elevated levels. Such young stars are encircled by molecular gas-rich disks, and the net removal of CRs from the circumstellar environment significantly reduces the expected CR ionization rate in the disk gas, most likely by many orders-of-magnitude. The loss of ionization reduces disk turbulence, and thereby affects both planet-formation and active chemical processes in the disk. We present models of CR exclusion and explore the implications for turbulence and for predicted chemical abundances. We also discuss means by which ALMA can be used to search for extrasolar heliosphere-analogs around young stars.
\end{abstract}

Cosmic Rays and the InterStellar Medium - CRISM 2014,

24-27 June 2014

Montpellier, France

\footnotetext{
${ }^{*}$ Speaker.
} 


\section{Introduction}

Ionization is fundamental to the chemical properties and physical structure of planet-forming disks around young stars. The gas-rich disks around T Tauri stars, analogues or the solar system at an age of a few Myr, experience a diversity of ionization sources, both in magnitude and spatial scope. Figure 1 illustrates the main disk ionizing agents, along with characteristic values. In the densest gas, the most important sources are stellar X-rays, short-lived radionuclide decay, and cosmic rays. UV radiation from the central star or the potentially enhanced local UV environment (the interstellar radiation field) may also contribute to the overall disk ionization but is fairly restricted to an outer surface due to efficient attenuation of UV light by small dust particles. We have observational handles for many of these components: $\mathrm{T}$ Tauri stars are measured to be $\mathrm{X}$-ray luminous, typically $L_{\mathrm{XR}} \sim 10^{28}-10^{31} \mathrm{erg} \mathrm{s}^{-1}[14]$ and are often time-variable, brightening by factors of a few. We can look towards the meteoritic record for evidence of our own short-lived radionuclide abundance history, where ${ }^{26} \mathrm{Al}$ was the dominant contributor towards SLR-derived ionization for the first few million years of the gas disk lifetime [29, 1, 11]. However, we have relatively little information regarding cosmic ray ionization of protoplanetary disks. Existing observations indicate that the CR ionization rate is not high, with limits of $\zeta_{\mathrm{CR}}<3 \times 10^{-17} \mathrm{~s}^{-1}$ determined from the non-detection of the dense gas ion-tracer $\mathrm{H}_{2} \mathrm{D}^{+}$[5].

There is some theoretical expectation that the cosmic ray ionization rate should be low. Cosmic rays are easily deflected by large scale interstellar or local disk magnetic fields $[12,7,24,13]$, or magnetized winds from the disk and/or the star itself [16, 6, 28, 7]. In this contribution, we present models of elevated levels of stellar wind modulation of cosmic rays by young $\mathrm{T}$ Tauri stars. The region over which winds operate is analogous to our own heliosphere, i.e., a "T Tauriosphere." We place these prescriptions in context with other contributors to the dense gas ionization, namely $\mathrm{X}$-rays and short-lived radionuclide decay [8]. We then make use of the sensitive link between the spatial structure of disk ionization and the chemistry of molecular ions to provide observational predictions. Deep submillimeter observations of molecular ions will help determine both the global ionization rate and potentially disentangle individual ionizing sources with spatial information. In this context, young stars with gas rich disks may provide the best opportunity to detect and probe the structure of extrasolar heliospheres, using the molecular content of the disk as a "test particle." Finally, we present applications of our model to the well-studied TW Hya protoplanetary disk system, where we have identified for the first time a substantially reduced CR rate in the circumstellar environment of another (in this case, young) star.

\section{Cosmic Ray Modulation by Winds}

The modern day solar wind, with a relatively modest mass loss rate $\left(\dot{M} \sim 10^{-14} \mathrm{M}_{\odot} \mathrm{yr}^{-1}\right)$ and typically Gauss-strength surface magnetic fields, drives out low energy CRs $\left(E_{\mathrm{CR}}<100 \mathrm{MeV}\right)$ from a $\sim 120$ AU region encompassing the solar system [34]. We can estimate the corresponding cosmic ray ionization rate for molecular hydrogen under modern day solar conditions by taking the measured cosmic ray proton spectra at heliocentric distances of $\sim 1 \mathrm{AU}$ and the energy-dependent $\mathrm{H}_{2}$ ionization cross section [23]. Under the influence of relatively mild sun-like winds, the cosmic ray ionization rate drops by a substantial level, $\sim 1-2$ orders of magnitude. In the environment 


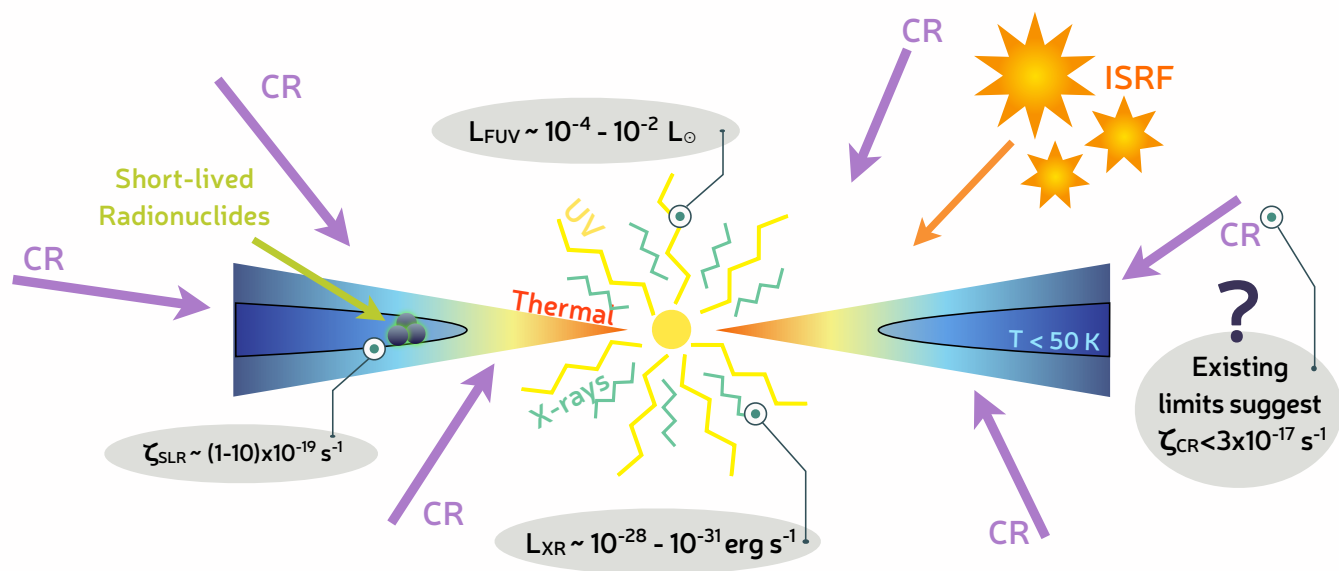

Figure 1: Sources of energetic ionization available to disks. The central star is both UV and X-ray bright. The FUV photons both from the central star as well as neighboring stars (the interstellar radiation field or ISRF) ionize species with low ionization potentials, such as C, S, and Si. X-rays from the star directly ionize $\mathrm{H}_{2}$ and $\mathrm{He}$, but primarily in the upper layers that are directly exposed to the X-rays. Short-lived radionuclides are locked up within the dust and thus ionize the disk internally, but their influence gradually decays over time. Cosmic rays (CR) are thought to pervade the outer disk since they experience the least obstruction. Observational evidence for each of these sources is highlighted in grey. All sources except the cosmic ray ionization rate have been measured in disks, whereas cosmic rays have only upper limits.

of a young star, with kilo-Gauss stellar magnetic fields [3, 20, 35] and measured mass loss rates typically $10 \%$ of the mass accretion rate [19], or $\dot{M} \lesssim 10^{-9} \mathrm{M}_{\odot} \mathrm{yr}^{-1}$ (i.e., five orders of magnitude higher than that of the sun) cosmic ray modulation should be even more severe. This realization motivated us to explore models under more extreme wind-modulation conditions, as would be expected for the circumstellar environment of young, magnetically active stars. To estimate the effects of enhanced young stellar winds, we start from the force-field approximation for the solar cosmic ray spectrum [18]. This approximation is an empirically derived prescription that describes the overall shape of the interplanetary cosmic ray spectrum to high accuracy with a single free parameter, the modulation potential [31]. The modulation potential has units of energy and conceptually describes the "barrier" due to winds that impedes the propagation of cosmic rays. The barrier varies in strength substantially over the solar magnetic activity cycle, with values of $500 \mathrm{MeV}$ to $1,500 \mathrm{MeV}$ [31]. To approximately estimate the enhanced level of wind-driven exclusion by a T Tauri star, we extrapolate these observations using the magnetic activity of the sun (traced by sunspot coverage) as our proxy. At the relatively high levels of magnetic activity typical of Myr-old stars, the local cosmic ray ionization flux is strongly modulated such that the ionization rate drops by an additional $\sim 1-2$ orders of magnitude below solar rates, resulting in net cosmic ray ionization rates of just $\zeta_{\mathrm{CR}} \sim 10^{-22}-10^{-20} \mathrm{~s}^{-1}$. Our simple extrapolated-modulation models agree with cosmic ray proton spectra from the literature for the Gyr-old sun [27, 10], which may imply that our estimates for the magnitude of wind-modulation by $\mathrm{T}$ Tauri stars are too conservative, and that the actual reduction of cosmic ray flux by winds may be more extreme. The full range of wind-modulated cosmic ray spectra are shown in Figure 2, where the diversity of local interstellar spectra (LIS) of cosmic rays highlights the difficulty of determining the particle spectrum at low 


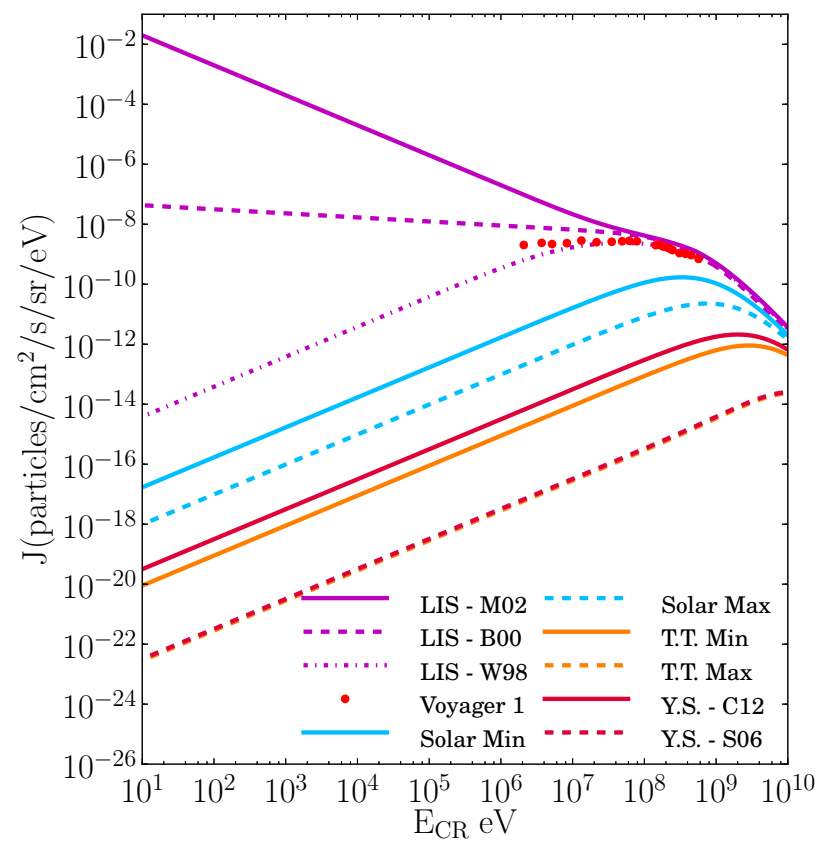

Figure 2: Cosmic ray proton spectra under the influence of stellar winds. The local interstellar spectra (LIS) are not well constrained below $1 \mathrm{MeV}$, as a direct result of the solar wind. The Voyager 1 points in red are the most recent direct measurements of the LIS [34]. The solar wind (blue) reduces the low energy end of the cosmic ray spectrum by over four orders of magnitude below $10 \mathrm{MeV}$. The orange lines show our extrapolated model sun results, estimated for T Tauri stars. These results agree with the relatively late-stage Gyr-old young sun models in red, indicating that the T Tauri spectra are possibly too high. Reproduced from Cleeves, Adams, and Bergin 2013, ApJ, 772, 5.

$(\lesssim 100 \mathrm{MeV}$ ) energies. The most important feature of the highly modulated T Tauri CR spectra (TT Min and TT Max, shown in orange), is that CRs of all energies, even at $\sim \mathrm{GeV}$, are deflected in the presence of extreme winds.

Cosmic ray exclusion by winds from young stars should be extreme. However, the region of influence affected by magnetized winds is unknown. If stellar winds are the source of the cosmicray deflection, then the winds may be diverted by the massive gas disk or be confined by stellar and/or disk magnetic fields, forming a doubly-lobed $\mathrm{T}$ Tauriosphere. This geometry would still permit cosmic ray ionization at the outer exposed rim of the disk. Alternatively, if large-scale, magnetized disk winds (e.g., centrifugally launched or photoevaporative winds) are able to drive out cosmic rays, they may create a larger shielded region. For example, many of the proplyds in Orion show wide-angle bow shocks not associated with a jet. These bow shocks have been attributed to a photoevaporative flow from the disk (greatly enhanced by UV irradiation from nearby massive stars) colliding with the stellar winds emanating from the neighboring massive young stars. In the case of proplyd 180-311, the bow shock wraps around the propyld at a projected radial distance of $700 \mathrm{AU}$ [17], encompassing roughly 200 times the volume of our own heliosphere. If such flows are able to modulate the cosmic ray flux similarly to the solar wind, they could fully enclose the disk and shield the entire disk from low energy cosmic rays. 


\section{Alternative Sources of Disk Ionization}

In the absence of cosmic rays, X-rays and short-lived radionuclides are alternative sources of $\mathrm{H}_{2}$ and He ionization in the very dense $\left(n_{\mathrm{H}_{2}} \sim 10^{10} \mathrm{~cm}^{-3}\right)$ gas. The ionization energy available in $\mathrm{X}$-rays is extremely large; however, the geometry of the star/disk system is such that the $\lesssim 3 \mathrm{keV} \mathrm{X}$ rays experience the highest levels of disk gas and dust absorption and are thus unable to efficiently penetrate the bulk gas. Thus the power in X-rays is mitigated by the large absorption column, which is the greatest at the inner disk near the central star, providing a "shield" for the outer disk. X-rays have the greatest influence in a surface layer where the gas surface density is below $\Sigma_{g} \lesssim 1 \mathrm{~g} \mathrm{~cm}^{-2}$ [4]. During stellar X-ray flares, however, the overall spectrum tends to harden, with relatively more energy emitted at $>3 \mathrm{keV}$ [26]. The harder X-ray photons are less efficiently stopped by the disk and are able to ionize more of the bulk disk mass. The relative importance of these time-dependent effects will depend on the strength and frequency of the stellar flares, as well as ion-recombination timescales within the disk.

Short-lived radionuclides provide a baseline to the dense gas ionization rate in the absence of cosmic rays; however, the typical rates range between $\zeta_{\mathrm{SLR}} \sim 10^{-18} \mathrm{~s}^{-1}$ in the inner disk to $\zeta_{\mathrm{SLR}} \sim 10^{-19} \mathrm{~s}^{-1}$ in the outer disk, taking into account the loss of the energetic decay products from the lower density regions of the disk [8]. This rate, furthermore, decays over timescales shorter than the disk lifetime, with a half-life of approximately 1.2 Myr. After $10 \mathrm{Myr}$ (without late-stage resupply of radioactive materials via supernovae or Wolf-Rayet stars [21, 22, 2]), the decay rate is just $\sim 0.3 \%$ of its initial value, or $\zeta_{\text {SLR }} \sim(1-10) \times 10^{-22} \mathrm{~s}^{-1}$.

\section{The Chemistry of Disk Ionization}

In light of these effects, it is clear that the global picture of disk ionization is highly complex. Winds and magnetic fields introduce orders of magnitude reduction in interstellar cosmic ray rates. X-rays provide a floor to the ionization rate of about $\zeta_{\mathrm{XR}}(1-10) \times \sim 10^{-21} \mathrm{~s}^{-1}$ (varying with disk mass), but hard X-ray flares enable deeper X-ray propagation, which can increase the instantaneous X-ray ionization rate by orders of magnitude during the flare. Likewise, the inherent time-dependence (and supply history) of the abundance of short-lived radionuclides like ${ }^{26} \mathrm{Al}$ also introduces at least an order of magnitude uncertainty in its contribution to the overall disk ionization budget [15, 30, 8].

Fortunately, the chemistry of molecular ions is highly sensitive to the underlying ionization environment. We explored a large grid of ionization models to calibrate the sensitivity of specific molecules and particularly specific emission lines to variations in the ionization source and magnitude [9]. Examples of the dependency are shown in Figure 3. $\mathrm{HCO}^{+}$traces very high cosmic ray ionization rates, but is insensitive to low rates once the cosmic ray contribution falls below the $\mathrm{X}$ ray contribution in the regions where $\mathrm{HCO}^{+}$is formed. $\mathrm{N}_{2} \mathrm{H}^{+}$and $\mathrm{N}_{2} \mathrm{D}^{+}$are particularly sensitive tracers of the deep ionization since these species are preferentially destroyed in warm gas, above $20 \mathrm{~K}$, due to $\mathrm{CO}$ evaporation.

We applied these results to one of the most extensively studied protoplanetary disks, TW Hya. TW Hya is relatively close $(d=55 \pm 9 \mathrm{pc})[33,32]$ and face on with an inclination of only $i \sim 7 \pm 1^{\circ}$ [25]. Starting from a well-calibrated model of the bulk gas disk, we explored twenty different ion- 


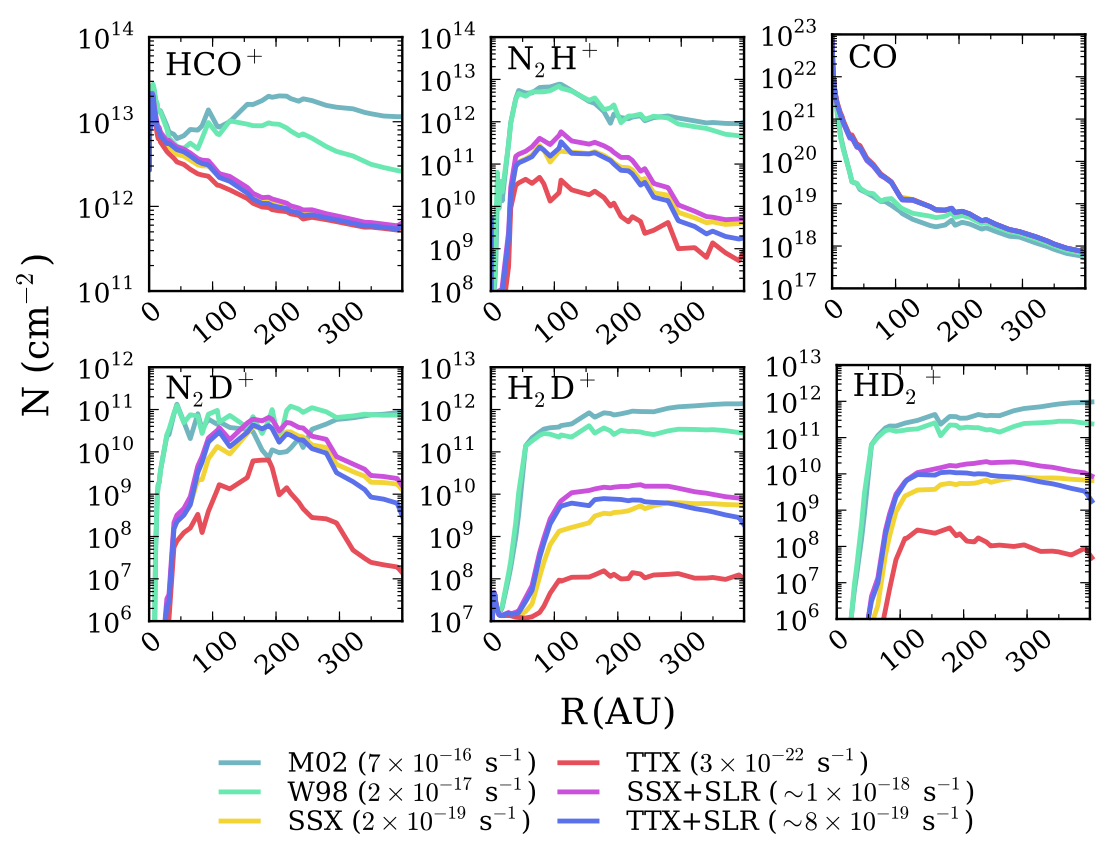

Figure 3: Variations in the column densities of important molecules for different cosmic ray ionization rates, with and without short-lived radionuclide ionization for the lowest CR ionization cases. These chemical signatures can be used in conjunction with observations to infer the ionization rate in Tauri systems. Adapted from Cleeves, Bergin, and Adams 2014, ApJ, 794, 123.

ization model combinations. We primarily varied the X-ray spectral hardness ratio (corresponding to small changes in X-ray luminosity, less than a factor of $\sim 3$ ) and the incident cosmic ray flux using the wind models described above. For each model combination, we calculated the resulting disk chemical structure, and then simulated the emergent line emission for lines of $\mathrm{HCO}^{+}$, $\mathrm{H}^{13} \mathrm{CO}^{+}$, and $\mathrm{N}_{2} \mathrm{H}^{+}$, comparing seven independent observations of molecular ion lines in total. The data were systematically fit better with low cosmic ray ionization rates, with the best fit models falling below $\zeta_{\mathrm{CR}} \lesssim 10^{-19} \mathrm{~s}^{-1}$. These results indicate that a significant degree of cosmic ray modulation is active in TW Hya, suppressing the overall ionization rate by two orders of magnitude or perhaps more. The actual cosmic ray rate could actually be much lower, because the measured rate appears to reflect TW Hya's stellar X-ray ionization floor. These results put strong limits on the combined cosmic ray and short-lived radionuclide ionization rates in the TW Hya protoplanetary disk. If winds are the primary driver of cosmic ray exclusion, these results may highlight the first detection of an external heliosphere, extending beyond $200 \mathrm{AU}$, i.e., beyond the molecular gas disk.

\section{Conclusions}

We have presented models that produce highly modulated cosmic ray ionization rates due to the influence of stellar winds from a young, Sun-like (T Tauri) star. We put the derived ionization rates into context by comparing with those of X-rays and radionuclides. Variations in all sources of ionization induce observational signatures in the molecular ion chemistry of disks, in particular, 
in the radial abundance profiles. Applying this knowledge, we have determined a substantially reduced cosmic ray ionization rate in TW Hya (Cleeves et al. 2014, submitted). In this particular system, the ionization appears to be primarily X-ray driven, implying that it may be difficult to further isolate the short-lived radionuclide and cosmic ray components without observations of the X-ray shielded gas in the inner disk, which will soon be possible with the full resolution and sensitivity of the Atacama Large Millimeter Array. With future observations and theoretical development, we will be able to measure the chemical structure of $\mathrm{T}$ Tauri disks, infer their ionization rates, and constrain the initial conditions for planet formation.

\section{References}

[1] F. C. Adams. The Birth Environment of the Solar System. ARA\&A, 48:47-85, September 2010.

[2] F. C. Adams, M. Fatuzzo, and L. Holden. Distributions of Short-lived Radioactive Nuclei Produced by Young Embedded Star Clusters. ApJ, 789:86, July 2014.

[3] G. Basri, G. W. Marcy, and J. A. Valenti. Limits on the magnetic flux of pre-main-sequence stars. ApJ, 390:622-633, May 1992.

[4] T. J. Bethell and E. A. Bergin. Photoelectric Cross-sections of Gas and Dust in Protoplanetary Disks. ApJ, 740:7, October 2011.

[5] E. Chapillon, B. Parise, S. Guilloteau, and F. Du. A deep search for $\mathrm{H}_{2} \mathrm{D}^{+}$in protoplanetary disks. Perspectives for ALMA. A\&A, 533:A143, September 2011.

[6] E. Chiang and R. Murray-Clay. Inside-out evacuation of transitional protoplanetary discs by the magneto-rotational instability. Nature Physics, 3:604-608, September 2007.

[7] L. I. Cleeves, F. C. Adams, and E. A. Bergin. Exclusion of Cosmic Rays in Protoplanetary Disks: Stellar and Magnetic Effects. ApJ, 772:5, July 2013.

[8] L. I. Cleeves, F. C. Adams, E. A. Bergin, and R. Visser. Radionuclide Ionization in Protoplanetary Disks: Calculations of Decay Product Radiative Transfer. ApJ, 777:28, November 2013.

[9] L. I. Cleeves, E. A. Bergin, and F. C. Adams. Exclusion of Cosmic Rays in Protoplanetary Disks. II. Chemical Gradients and Observational Signatures. ApJ, 794:123, October 2014.

[10] O. Cohen, J. J. Drake, and J. Kóta. The Cosmic-Ray Intensity near the Archean Earth. ApJ, 760:85, November 2012.

[11] N. Dauphas and M. Chaussidon. A Perspective from Extinct Radionuclides on a Young Stellar Object: The Sun and Its Accretion Disk. Annual Review of Earth and Planetary Sciences, 39:351-386, May 2011.

[12] A. Z. Dolginov and T. F. Stepinski. Are cosmic rays effective for ionization of protoplanetary disks? ApJ, 427:377-383, May 1994.

[13] M. Fatuzzo and F. C. Adams. Effects of Turbulence on Cosmic Ray Propagation in Protostars and Young Star/Disk Systems. ApJ, 787:26, May 2014.

[14] E. D. Feigelson, P. Broos, J. A. Gaffney, III, G. Garmire, L. A. Hillenbrand, S. H. Pravdo, L. Townsley, and Y. Tsuboi. X-Ray-emitting Young Stars in the Orion Nebula. ApJ, 574:258-292, July 2002.

[15] F. Finocchi and H.-P. Gail. Chemical reactions in protoplanetary accretion disks. III. The role of ionisation processes. A\&A, 327:825-844, November 1997. 
[16] C. F. Gammie. Layered Accretion in T Tauri Disks. ApJ, 457:355, January 1996.

[17] F. García-Arredondo, W. J. Henney, and S. J. Arthur. Hydrodynamic Simulations of Proplyd Bow Shocks. ApJ, 561:830-842, November 2001.

[18] L. J. Gleeson and W. I. Axford. Cosmic Rays in the Interplanetary Medium. ApJ, 149:L115, September 1967.

[19] P. Hartigan, S. Edwards, and L. Ghandour. Disk Accretion and Mass Loss from Young Stars. ApJ, 452:736, October 1995.

[20] C. M. Johns-Krull, J. A. Valenti, and C. Koresko. Measuring the Magnetic Field on the Classical T Tauri Star BP Tauri. ApJ, 516:900-915, May 1999.

[21] N. Ouellette, S. J. Desch, and J. J. Hester. Interaction of Supernova Ejecta with Nearby Protoplanetary Disks. ApJ, 662:1268-1281, June 2007.

[22] N. Ouellette, S. J. Desch, and J. J. Hester. Injection of Supernova Dust in Nearby Protoplanetary Disks. ApJ, 711:597-612, March 2010.

[23] M. Padovani and D. Galli. Effects of magnetic fields on the cosmic-ray ionization of molecular cloud cores. A\&A, 530:A109, June 2011.

[24] M. Padovani, P. Hennebelle, and D. Galli. Cosmic-ray ionisation in collapsing clouds. $A \& A$, 560:A114, December 2013.

[25] C. Qi, P. T. P. Ho, D. J. Wilner, S. Takakuwa, N. Hirano, N. Ohashi, T. L. Bourke, Q. Zhang, G. A. Blake, M. Hogerheijde, M. Saito, M. Choi, and J. Yang. Imaging the Disk around TW Hydrae with the Submillimeter Array. ApJ, 616:L11-L14, November 2004.

[26] S. L. Skinner, M. Guedel, K. Koyama, and S. Yamauchi. ASCA Observations of the Barnard 209 Dark Cloud and an Intense X-Ray Flare on V773 Tauri. ApJ, 486:886, September 1997.

[27] H. Svensmark. Cosmic rays and the biosphere over 4 billion years. Astronomische Nachrichten, 327:871, November 2006.

[28] N. J. Turner, T. Sano, and N. Dziourkevitch. Turbulent Mixing and the Dead Zone in Protostellar Disks. ApJ, 659:729-737, April 2007.

[29] T. Umebayashi and T. Nakano. Fluxes of Energetic Particles and the Ionization Rate in Very Dense Interstellar Clouds. PASJ, 33:617-+, 1981.

[30] T. Umebayashi and T. Nakano. Effects of Radionuclides on the Ionization State of Protoplanetary Disks and Dense Cloud Cores. ApJ, 690:69-81, January 2009.

[31] I. G. Usoskin, K. Alanko-Huotari, G. A. Kovaltsov, and K. Mursula. Heliospheric modulation of cosmic rays: Monthly reconstruction for 1951-2004. Journal of Geophysical Research (Space Physics), 110:12108, December 2005.

[32] F. van Leeuwen. Validation of the new Hipparcos reduction. A\&A, 474:653-664, November 2007.

[33] R. A. Webb, B. Zuckerman, I. Platais, J. Patience, R. J. White, M. J. Schwartz, and C. McCarthy. Discovery of Seven T Tauri Stars and a Brown Dwarf Candidatein the Nearby TW Hydrae Association. ApJ, 512:L63-L67, February 1999.

[34] W. R. Webber and F. B. McDonald. Recent Voyager 1 data indicate that on 25 August 2012 at a distance of 121.7 AU from the Sun, sudden and unprecedented intensity changes were observed in anomalous and galactic cosmic rays. Geophys. Res. Lett., 40:1665-1668, May 2013.

[35] H. Yang, C. M. Johns-Krull, and J. A. Valenti. Measuring the Magnetic Field of the Classical T Tauri Star TW Hydrae. ApJ, 635:466-475, December 2005. 